\title{
Brief advice reduced drinking in non-dependent problem drinkers
}

Fleming MF, Barry KL, Manwell LB, et al. Brief physician advice for problem alcohol drinkers. A randomized controlled trial in communitybased primary care practices. JAMA 1997 Apr 2;277:1039-45.

\section{Objective}

To determine the effectiveness of brief advice given by primary care physicians to problem drinkers.

\section{Design}

1 year randomised controlled trial (Trial for Early Alcohol Treatment $[\mathrm{TrEAT}])$.

\section{Setting}

17 community clinics in Wisconsin, USA, comprising primary care practices of 64 general internists and family physicians.

\section{Patients}

Of 17695 patients screened, 2925 had a drinking problem and 1705 were interviewed by a researcher to determine eligibility. Screening yielded 774 patients who were 18 to 65 years of age $(62 \%$ men) and drank $>14$ alcoholic drinks/week (11 drinks/wk for women). Exclusion criteria were pregnancy, alcohol treatment in the previous year, symptoms of alcohol withdrawal in the past 12 months, receipt of physician advice to change alcohol use in the past 3 months, consumption of $>50$ drinks/week, or reported symptoms of suicide. 12 month follow up was $93 \%$.

\section{Intervention}

Patients were allocated to a brief physician advice intervention group $(n=392)$ or to a control group $(n=382)$. Patients allocated to the intervention group had two 15 minute sessions with their physician 1 month apart, during which they received feedback about their current health behaviours, a review of the prevalence of problem drinking and adverse effects, a list of drinking cues, a drinking agreement, and drinking diary cards. A follow up telephone call from the clinic nurse was made 2 weeks after each visit. Patients in the control group received a booklet on general health issues.

\section{Main outcome measures}

Changes in alcohol use (previous weekly use, binge drinking, and excessive drinking), health care utilisation, and changes in health status measures (smoking status, depression, motor vehicle accidents, and unintentional injuries) at 6 and 12 months.

\section{Main results}

Reductions in alcohol use were greater in patients who received the intervention than in patients in the control group. At 12 months, the mean number of drinks in the previous week was 11.48 in patients who received the intervention compared with 15.46 in patients in the control group $(\mathrm{p}<0.001)$. The number of binge-drinking episodes in the previous month also decreased to a greater extent in the intervention group (46\% reduction) than in the control group (21\% reduction). Patients who received the intervention reported fewer days of hospitalisation (91 $v 146 \mathrm{~d}, \mathrm{p}<0.001)$. Changes in health status did not differ between groups.

\section{Conclusions}

An intervention programme involving brief physician advice to non-dependent problem drinkers reduced alcohol use. The reduction was maintained at 12 months.

Source of funding: National Institute on Alcohol Abuse and Alcoholism.

For article reprint:Dr M Fleming, 777 South Mills Street, Madison, WI 53715, USA. Fax +1 608 2635813.

Abstract and commentary also published in ACP Journal Club 1977 Nov-Dec;127:63 and Evidence-Based Medicine 1997 Nov-Dec;2:183.

\section{Commentary}

Because of its greater prevalence, heavy drinking and not alcohol dependence is responsible for most alcohol related morbidity. ${ }^{1}$ Thus, recent attention has turned to non-dependent drinkers. Evidence shows that brief interventions can decrease alcohol use in problem drinkers. $^{2}$

The study by Fleming et al extends previous findings to community primary care settings in the United States. 2 limitations may have caused overestimates of efficacy. Firstly, patients rather than physicians were randomly assigned; secondly, greater losses to follow up (likely nonresponders) occurred in the intervention group. However, the intention to treat analysis and the consistency of the results with those of previous studies support conclusions of efficacy. ${ }^{2}$
The challenge is how to incorporate brief interventions into practice. In this study, more than half of the problem drinkers initially identified by routine screening were excluded after a research interview; how they would have responded to the intervention is unknown. Furthermore, the intervention was extensive, including 2 physician visits and follow up calls, written materials, payments, and ongoing physician education. Finally, most patients continued to binge drink and, although days of hospitalisation were decreased, no decreases in injures or emergency department visits were found and the clinical implications of a decrease to 12 drinks per week are unclear.

When attention is given to identifying problem drinkers or those at risk who are not alcohol dependent, brief physician advice can reduce alcohol consumption; however, questions remain. How effective and efficient are brief interventions for improving health outcomes? How should patients be selected? How brief an intervention is effective? What minimum level of physician education and support is necessary? These issues must be addressed before this promising practice can be widely implemented.

Richard Saitz, MD, MPH
Boston University
Boston,
Massachusetts, USA
edicine. Broadening the base of
cohol problems. Washington DC:
emy Press, 1990.
cohol problems: a review. Addic-
$15-35$.

1 Institute of Medicine. Broadening the base of treatment for alcohol problems. Washington DC: Bien TH, Miller WR, Tonigan JS. Brief interventions for alcohol problems: a review. Addiction 1993;88:315-35. 